\title{
Avaliação do Intervalo Tpico-Tfim e QT em Doença de Chagas, Fase Crônica e Eletrocardiograma Normal
}

\section{Evaluation of Tpeak-Tend and QT intervals in Chagas' Disease, Chronic Phase and Normal Electrocardiogram}

\author{
Abilio Augusto Fragata Filho ${ }^{1}$, Claudia da Silva Fragata1, ${ }^{1}$, Angela Maria Lourenço ${ }^{1}$, \\ Cristiane Castro Faccini ${ }^{1}$, João Ítalo Dias França ${ }^{1}$
}

ORCID IDS

Fragata Filho AA (D) https://orcid.org/0000-0002-9322-3662

Fragata CS (D) https://orcid.org/0000-0002-4056-3380

Lourenço AM (D) https://orcid.org/0000-0002-8609-0347

\author{
Faccini CC (D) https://orcid.org/0000-0002-4056-3380 \\ França JID (D) https://orcid.org/0000-0001-5606-5960
}

\begin{abstract}
RESUMO
Introdução: A doença de Chagas carece de elementos que proporcionem prever quais portadores evoluirão para a forma cardíaca e quais permanecerão na forma indeterminada. Objetivo: Avaliar relação entre evolução eletrocardiográfica e intervalos Tpico-Tfim e QT, ambos corrigidos para a frequência cardíaca, em chagásicos com eletrocardiograma inicial normal. Método: Avaliamos retrospectivamente chagásicos admitidos na instituição até 2002, tendo eletrocardiograma normal na primeira consulta. Os intervalos Tpico-Tfim e QT foram medidos em milissegundos nas derivações V2 e V5, sendo a média dos valores corrigida para a frequência cardíaca (Bazett). Analisou-se a relação desses valores com a evolução eletrocardiográfica dos indivíduos, tendo em vista sexo, tempo de evolução e se receberam benzonidazol ou não. Resultados: O intervalo Tpico-Tfim corrigido não mostrou significância estatística entre os que mantiveram ou não eletrocardiograma normal. O intervalo QT corrigido, o tratamento com benzonidazol e o tempo de evolução mostraram significância quanto à manutenção do eletrocardiograma normal. $\mathrm{Na}$ avaliação multivariada, o tratamento com benzonidazol, o intervalo QTc e o tempo de evolução se mostraram variáveis independentes para a manutenção do eletrocardiograma normal. Conclusão: $O$ intervalo Tpico-Tfim não se mostrou preditor da evolução eletrocardiográfica. O intervalo QT aumentado favoreceu aparecimento de alterações.
\end{abstract}

PALAVRAS-CHAVE: Doença de Chagas; Intervalo TpicoTfim; Intervalo QT.

\begin{abstract}
Introduction: Chagas' disease lacks elements to predict which carriers will evolve into cardiac form and which will remain in undetermined form. Objective: To evaluate the relationship between electrocardiographic evolution and Tpeak-Tend and QT intervals, both corrected for heart rate, in chagasic patients with normal initial electrocardiogram. Method: Chagasic patients admitted to the institution until 2002 were retrospectively evaluated, and the electrocardiogram was normal at the first consultation. The Tpeak-Tend and QT intervals were measured in milliseconds at the V2 and V5 derivations, with the mean values corrected for heart rate (Bazett). The relationship of these values with the electrocardiographic evolution of the individuals was analyzed, considering the gender, time of evolution and whether they received benznidazole or not. Results: The corrected Tpeak-Tend interval showed no statistical significance among those who maintained or not normal electrocardiogram. The corrected QT interval, the treatment with benznidazole and the time of evolution showed significance for the maintenance of normal electrocardiogram. In the multivariate evaluation, treatment with benznidazole, the QTC interval and the time of evolution were independent variables for the maintenance of normal electrocardiogram. Conclusion: The Tpeak-Tend interval showed no predictor of electrocardiographic evolution. The increased QT interval favored changes.
\end{abstract}

KEYWORDS: Chagas' disease; Tpeak-Tend interval; QT interval.

1. Instituto Dante Pazzanese de Cardiologia - Laboratório de Doença de Chagas - São Paulo (SP), Brasil

Recebido: Jan. 16, 2020 | Aceito: Jan. 30, 2019

*Autora correspondente: claudiadasilvafragata@gmail.com

Editor de seção: José Tarciso Medeiros de Vasconcelos

Esta pesquisa foi aprovada pelo Comitê de Ética em Pesquisa do Instituto Dante Pazzanese de Cardiologia de São Paulo sob o número 006624/2017. 


\section{INTRODUÇÃO}

A doença descrita por Carlos Chagas em 1909 ainda é um grave problema de saúde pública, não só na América Latina como também em vários países não endêmicos. Portadores do parasita, sabidos ou não, migraram para os diversos continentes, transmitindo essa doença através da transfusão de sangue ou doação de órgãos, fazendo com que essa enfermidade seja encontrada atualmente em vários outros continentes ${ }^{1}$.

Após a fase aguda, inicia-se a fase crônica e, inicialmente, não há manifestações clínicas, mas somente sorologia positiva (forma indeterminada: período da fase crônica que pode se estender por toda a vida do paciente, caracterizado por sorologia positiva - duas técnicas diferentes - ausência de sintomas, eletrocardiograma de superfície, radiografia de tórax, esofagograma e enema opaco normais). $\mathrm{Na}$ fase crônica tardia, cerca de $40 \%$ dos acometidos desenvolve doença cardíaca, que se manifesta por arritmias, insuficiência cardíaca, fenômenos tromboembólicos e/ou morte súbita, que pode ocorrer independentemente da presença de sintomas, constituindo-se em motivo de grande preocupação. Não se conhece até hoje marcador que permita saber quais serão os pacientes que irão desenvolver cardiopatia e quais permanecerão em forma indeterminada por toda sua vida ${ }^{1}$.

A análise da repolarização ventricular, principalmente o intervalo QT e o intervalo entre o pico da onda $\mathrm{T}$ e seu final (Tpico-Tfim), tem interessado pesquisadores, haja vista as evidências de sua importância na estratificação de risco para o desenvolvimento de arritmias graves e morte súbita ${ }^{2-6}$.

Em portadores de disfunção sistólica de ventrículo esquerdo, isquêmicos e não isquêmicos, demonstrou-se que o prolongamento do intervalo Tpico-Tfim foi preditor independente de taquiarritmias ventriculares e mortalidade geral $^{3}$. Na presença de embolia pulmonar, também se verificou que Tpico-Tfim alargado foi marcador precoce de má evolução ${ }^{4}$. Quando do intervalo QT corrigido (QTc) normal, a presença de alargamento do Tpico-Tfim foi marcador independente associado à morte súbita ${ }^{5}$.

$\mathrm{Na}$ doença de Chagas, os estudos relacionados aos parâmetros de repolarização ventricular são poucos e dizem respeito à presença de cardiopatia chagásica crônica instalada e não a pacientes com eletrocardiograma normal. Em pacientes chagásicos, havendo bloqueio do ramo direito, associado ou não ao bloqueio do fascículo anterossuperior esquerdo do feixe de His, a duração do Tpico-Tfim e do
QT relacionaram-se com aumento da mortalidade ou da necessidade de transplante cardíaco ${ }^{6,7}$. Igualmente em pacientes com cardiopatia chagásica, o estudo eletrofisiológico induzindo arritmias ventriculares por estimulação ventricular programada mostrou modesta relação (não significativa) com o alargamento de Tpico-Tfim ${ }^{8}$.

Tendo em vista a escassez de dados disponíveis na literatura, a análise de parâmetros da atividade elétrica ventricular em pacientes com doença de Chagas, fase crônica e eletrocardiograma normal, faz-se necessária, buscando possíveis elementos que permitam relacionar à evolução clínica desses pacientes.

\section{OBJETIVOS}

O objetivo deste artigo é avaliar retrospectivamente a relação entre a evolução eletrocardiográfica e a média dos valores dos intervalos Tpico-Tfim e QT (corrigidos pela frequência cardíaca) medidos no eletrocardiograma convencional normal em pacientes com doença de Chagas, fase crônica, tratados ou não com benzonidazol.

\section{METODOLOGIA}

Os prontuários de pacientes portadores de doença de Chagas confirmada por duas ou mais técnicas de reação de soro foram analisados do banco de dados do Laboratório de Doença de Chagas do Instituto Dante Pazzanese de Cardiologia.

Os critérios de inclusão foram: 1) estar em fase crônica, admitidos na instituição em 2002 ou antes; 2) eletrocardiograma convencional normal na primeira consulta (velocidade de $25 \mathrm{~mm} / \mathrm{s}$ e amplitude de $1 \mathrm{mv} / \mathrm{mm}$ ); 3) tratados ou não com benzonidazol; 4) ambos os sexos; 5) sem restrição de idade. Não houve critérios de exclusão.

Em nenhum paciente foi realizado esofagograma ou enema opaco, razão pela qual eles não são referidos neste artigo com forma indeterminada, mas sim como pacientes chagásicos e eletrocardiograma normal.

As derivações $\mathrm{V} 2 \mathrm{e} \mathrm{V} 5$ do eletrocardiograma convencional foram utilizadas, digitalizadas e ampliadas para perfeita visibilização e aferição manual das medidas de tempo:TpicoTfim e QT, sendo então obtida a média desses valores (Fig.1). Foram obtidas também as medidas: (Fig. 1a) frequência 
(a)

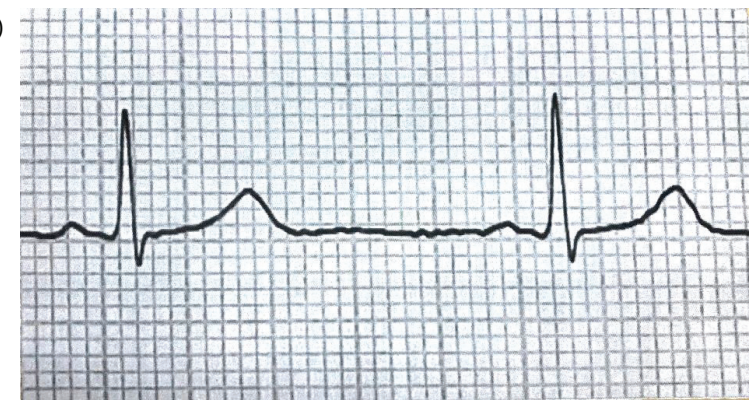

(b)

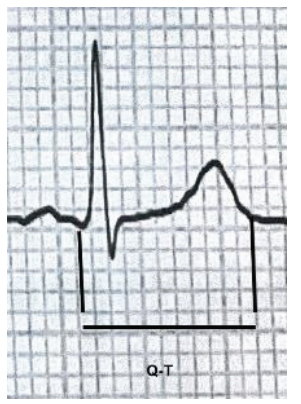

(c)

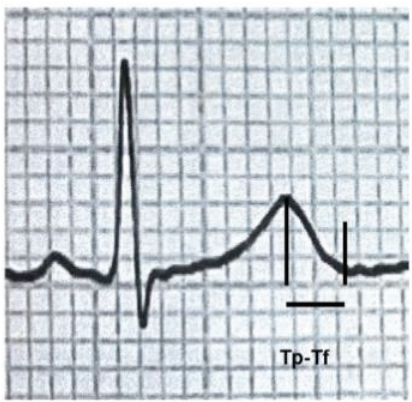

Figura 1. Eletrocardiograma convencional ampliado.

cardíaca (FC) medida em batimentos por minuto (bpm); (Fig. 1b) intervalo Tpico-Tfim corrigido (Tp-Tf)c para FC em milissegundos (ms); (Fig. 1c) intervalo QT corrigido para frequência cardíaca (QTc) em ms.

A ocorrência de cardiopatia (caracterizada pelo aparecimento de alterações eletrocardiográficas no último traçado do paciente) foi analisada, sendo o período mínimo de seguimento de 10 anos.

As médias aritméticas dos valores de QTc e (Tp-Tf) c foram relacionadas com: idade (média- desvio padrão) em anos; sexo (masculino-feminino); tratamento com benzonidazol (sim-não); tempo de evolução em anos; evolução para cardiopatia (sim-não). As diferentes etnias não foram analisadas por não ter sido feita verificação objetiva, mas sim dados coletados do prontuário.

A FC foi obtida pela divisão de $60.000 \mathrm{~ms}$ pelo intervalo R-R em milissegundos (60.000 ms / R-R ms). Quando os intervalos $\mathrm{R}-\mathrm{R}$ não fossem regulares, foi obtida a média de 3 batimentos. $O$ intervalo QT foi medido em ms do início do complexo QRS ao término da onda $\mathrm{T}$ (representando a duração total da atividade elétrica ventricular). O intervalo QTc foi obtido pela fórmula de Bazett, que consiste na divisão do intervalo QT, medido em $\mathrm{ms}$, pela raiz quadrada do intervalo $\mathrm{R}-\mathrm{R}$ igualmente em ms $(\mathrm{QTc}=\mathrm{QT} / \sqrt{\mathrm{R}-\mathrm{R}})$, fornecendo assim o valor do intervalo QT ajustado para a frequência cardíaca. Para frequências cardíacas menores que $60 \mathrm{bpm}$ ou superiores a $90 \mathrm{bpm}$, foram utilizadas fórmulas lineares, como as de Framingham:

QT correção linear $-\mathrm{QTcl}=\mathrm{QT}+$ 0,154 $(1-\mathrm{R}-\mathrm{R})$

e Hodges ${ }^{10,11}$ (Fig. 1b):

QT correção linear - QTm: QT + 1,75 (FC - 60)
$\mathrm{O}$ intervalo Tpico-Tfim foi medido em ms, entre o pico da onda $\mathrm{T}$ e seu final, corrigindo-se para a frequência cardíaca, utilizando-se a fórmula de Bazett, com substituição de QT por Tpico-Tfim (Fig. 1c).

A análise estatística foi realizada avaliando-se a relação das médias dos valores de QTc e (Tp-Tf)c com os parâmetros descritos, utilizando-se o software IBM SPSS versão 19 (ARMONK NY).

\section{RESULTADOS}

Foram analisados 291 pacientes que preenchiam os critérios de inclusão. Desses, 59 com FC $<60$ e $9>90 \mathrm{bpm}$. Usando-se a fórmula de Bazett para o cálculo do QTc, a média foi de 408,35 ms e, aplicando-se a fórmula de Hodges nos pacientes com $\mathrm{FC}<60 \mathrm{ou}$ > 90 bpm, a média do QTc foi de 406,85 ms, sem diferença significativa. A Tabela 1 mostra a análise univariada das características estudadas.

Nessa análise univariada, observou-se que a frequência cardíaca, o intervalo $\mathrm{Tp}$-Tf e ( $\mathrm{Tp}$-Tf $)$ c, a idade e o sexo não tiveram diferença estatisticamente significativa entre os pacientes que permaneceram com eletrocardiograma normal e aqueles cujo eletrocardiograma se alterou. O intervalo QT, o QTc e o tempo de evolução se mostraram maiores nos pacientes que tiveram seu eletrocardiograma alterado, enquanto que os pacientes tratados com benzonidazol permaneceram em maior número com eletrocardiograma normal, sendo estes três com diferenças estatisticamente significativas. A Tabela 2 mostra a análise multivariada da ocorrência de alterações do eletrocardiograma.

$\mathrm{Na}$ análise multivariada, considerando-se a alteração do eletrocardiograma, os pacientes tratados com benzonidazol tiveram porcentagem menor de alterações no traçado 
Tabela 1. Análise univariada da frequência cardíaca, QTc, (Tp-Tf)c, idade, sexo, tratamento com benzonidazol, tempo de evolução dos pacientes que mantiveram ECG normal ou não, com seguimento mínimo de 10 anos.

\begin{tabular}{|c|c|c|c|c|c|c|c|}
\hline \multirow{3}{*}{ Variável } & \multirow{2}{*}{\multicolumn{2}{|c|}{ Geral }} & \multicolumn{4}{|c|}{ ECG Normal } & \multirow{3}{*}{ P-valor } \\
\hline & & & \multicolumn{2}{|c|}{ Não } & \multicolumn{2}{|c|}{ Sim } & \\
\hline & $\begin{array}{l}\text { Número ou } \\
\text { média }\end{array}$ & $\begin{array}{c}\% \\
\text { ou D.P. }\end{array}$ & $\begin{array}{l}\text { Número ou } \\
\text { média }\end{array}$ & $\begin{array}{c}\% \\
\text { ou D.P. }\end{array}$ & $\begin{array}{l}\text { Número ou } \\
\text { média }\end{array}$ & $\begin{array}{c}\% \\
\text { ou D.P. }\end{array}$ & \\
\hline Frequência cardíaca & 67,2 & 11,1 & 65,5 & 9,6 & 67,8 & 11,5 & 0,1962 \\
\hline QT m & 378,97 & 35,28 & 388,53 & 35,29 & 375,65 & 34,74 & 0,0065 \\
\hline QTcm & 399,1 & 33,6 & 406,9 & 41,1 & 396,4 & 30,2 & 0,0383 \\
\hline (TpTf) m & 84,33 & 11,10 & 85,87 & 12,64 & 83,80 & 10,50 & 0,2624 \\
\hline (TpTf)c m & 89,2 & 13,2 & 90,0 & 14,9 & 88,9 & 12,5 & 0,7886 \\
\hline T. evolução & 19,6 & 6,4 & 22,3 & 6,5 & 18,7 & 6,1 & 0,0000 \\
\hline Idade & 38,2 & 10,2 & 38,3 & 9,9 & 38,1 & 10,3 & 0,9156 \\
\hline Sexo masculino & 101 & 34,8 & 24 & 32,0 & 77 & 35,8 & 0,5766 \\
\hline Tratamento & 248 & 85,2 & 53 & 70,7 & 195 & 90,3 & 0,0001 \\
\hline
\end{tabular}

Tabela 2. Análise Multivariada entre o ECG alterado e as variáveis Idade, Sexo, Tratamento com Benzonidazol, Tempo de evolução, e QTcm.

\begin{tabular}{ccccc}
\hline \multirow{2}{*}{ Variável } & \multirow{2}{*}{ Odds Ratio } & \multicolumn{2}{c}{ IC de 95\% } & \multirow{2}{*}{ P-valor } \\
\cline { 3 - 4 } & & Limite inferior & Limite superior & 0,9395 \\
\hline Idade & 1,001 & 0,967 & 1,037 & 0,7529 \\
\hline Sexo masculino & 1,106 & 0,590 & 2,075 & 0,0018 \\
\hline Benzonidazol & 0,262 & 0,113 & 0,607 & 0,0005 \\
\hline T. Evolução & 1,091 & 1,039 & 1,146 & 0,0297 \\
\hline QTcm & 1,010 & 1,001 & 1,019 & \\
\hline
\end{tabular}

eletrocardiográfico. Esses resultados já foram demonstrados anteriormente ${ }^{12}$; os pacientes com tempo de evolução mais prolongado apresentaram mais exames alterados. O QTc progressivamente maior, favoreceu o aparecimento de alterações do eletrocardiograma. Essas três variáveis se comportaram de forma independente.

\section{DISCUSSÃO}

A doença de Chagas em sua fase crônica se caracteriza pelas formas indeterminada e clínica, sendo que a primeira persiste indefinidamente em cerca de 60\% dos indivíduos afetados. Não há, até o presente, marcadores que permitam identificar quais pacientes manterão a forma indeterminada para sempre ou aqueles que, após período de tempo variável, evoluirão para a cardiopatia. A análise da repolarização ventricular tem se mostrado como importante valor prognóstico em várias cardiopatias ${ }^{13}$.

Os valores de $\mathrm{T}$ p-Tf acima de 100 ms são considerados anormais, havendo poucos dados disponíveis de valores corrigidos para $\mathrm{FC}^{4}$. Para os valores de QTc, sabe-se que variam com o sexo e são aceitos como normais até o máximo de $450 \mathrm{~ms}$ para homens e $470 \mathrm{~ms}$ para mulheres ${ }^{9}$.

Vários estudos têm ressaltado a importância dos parâmetros da atividade elétrica e da repolarização ventricular em várias doenças cardíacas com alterações eletrocardiográficas, mas não em doenças (como a doença de Chagas) com eletrocardiograma normal ${ }^{3-7}$.

Em avaliação de 272 pacientes com embolia pulmonar aguda, observou-se que os valores de Tp-Tf acima de $126 \mathrm{~ms}$ foram preditores de morbimortalidade, com sensibilidade de $80,56 \%$, especificidade de 59,32\%, valor preditivo negativo de $95,2 \%$ e valor preditivo positivo de $23,2 \%{ }^{4}$.

Analisando 50 pacientes com fluxo coronariano lento e 40 controles, os autores observaram aumento significativamente maior do ( $\mathrm{Tp}$-Tf ) e QTc naqueles com alterações da velocidade do fluxo nas coronárias, comparados aos controles ${ }^{14}$.

De 327 pacientes com fração de ejeção $\leq 35 \%$, portadores de cardiodesfibrilador implantável, observou-se que, em um período de $17 \pm 12$ meses, houve choques apropriados em 59 (18\%) e, em $30 \pm 13$ meses, 67 (21\%) morreram. O 
intervalo $\mathrm{T} p-\mathrm{Tf}$ foi variável independente, sendo que a cada aumento de $10 \mathrm{~ms}$, houve aumento de 1,16 nos choques apropriados e 1,14 na mortalidade por qualquer causa ${ }^{3}$.

Avaliando 695 pacientes com doença coronária, 353 com morte súbita (recuperada ou não), os autores observaram que o intervalo $\mathrm{T} p-\mathrm{Tf}$ medido na derivação V5 do eletrocardiograma convencional, mostrou-se como preditor desse evento, principalmente quando o intervalo QTc era normal ${ }^{5}$.

Em 105 pacientes com cardiopatia chagásica crônica (ECG anormal) submetidos a estudo eletrofisiológico e indução ou não de arritmia ventricular sustentada, observou-se que o intervalo $\mathrm{Tp}-\mathrm{Tf}$ maior que $100 \mathrm{~ms}$ mostrou tendência (p: 0,07) em identificar pacientes com maior risco de arritmias ventriculares ${ }^{8}$.

Acompanhando 738 pacientes cardiopatas chagásicos crônicos por $58 \pm 39$ meses, os autores observaram que a análise do intervalo QT, mas não do Tp-Tf, foi importante preditor de mortalidade nestes pacientes ${ }^{6}$.

No presente estudo, 291 pacientes chagásicos crônicos foram retrospectivamente avaliados, com eletrocardiograma normal na primeira consulta e sua evolução após cerca de duas décadas. O valor prognóstico do intervalo (Tp-Tf)c e do intervalo QTc foi avaliado e medido no eletrocardiograma normal da admissão, relacionando essas medidas com a manutenção do eletrocardiograma normal ou o aparecimento de quaisquer anormalidades no traçado eletrocardiográfico. Concordando com os dados da literatura, o intervalo $\mathrm{Tp}-\mathrm{Tf}$ e $(\mathrm{T} \mathrm{p}-\mathrm{Tf}) \mathrm{c}$ não se mostrou diferente entre os pacientes que se mantiveram com eletrocardiograma normal ou alterado, não sendo marcador de evolução eletrocardiográfica nos pacientes chagásicos, com eletrocardiograma normal.

Em estudo retrospectivo, com duas décadas de observação, a importância do tratamento parasiticida com benzonidazol na manutenção do eletrocardiograma normal foi demonstrada anteriormente ${ }^{12}$. Muito embora não fizesse parte do objetivo deste estudo, o resultado se manteve nesta avaliação, sendo que o tratamento parasiticida foi variável independente, favorecendo a manutenção do eletrocardiograma normal (diminuição de $75 \%$ de chances de haver alterações no traçado - Tabela 2).

O QTc e o tempo de evolução foram variáveis independentes, sendo que quanto maiores esses parâmetros, maiores as chances de haver alterações do eletrocardiograma. De acordo com a Tabela 2, a cada unidade a mais de QTc houve aumento de chance do traçado eletrocardiográfico se mostrar alterado de $0,9 \%$ e, a cada ano de evolução, essas chances foram de 9,1\%.

Baseando-se no critério de Youden15, o melhor ponto de corte, maximizando o acerto, seria QTc de 425,5 ms, o que proporcionaria uma sensibilidade de $37,3 \%$ e especificidade de $81,5 \%$. Como neste estudo a análise do QTc foi feita em pacientes com eletrocardiograma normal, os autores acreditam que a sensibilidade e não a especificidade deveria ser priorizada, haja vista estar-se buscando valores que possam indicar maior probabilidade de aparecimento de anormalidades eletrocardiográficas. Assim sendo, optou-se por nível de corte com sensibilidade de $80 \%$, o que corresponde ao valor QTc de $372 \mathrm{~ms}$, muito embora a especificidade seria de 21,3\% com esse valor. Seria mais apropriado identificar o maior número de pacientes com eletrocardiograma normal que teriam maior chance de apresentar alteração do traçado eletrocardiográfico, mesmo com a existência de muitos falsos positivos que seriam triados com outros métodos.

Dessa forma, a análise do intervalo $\mathrm{T} p-\mathrm{Tf}$ em pacientes com doença de Chagas, fase crônica e eletrocardiograma normal, não foi capaz de identificar pacientes que se manteriam ou não com traçado eletrocardiográfico normal. Por outro lado, o QTc acima de $372 \mathrm{~ms}$ se mostrou com sensibilidade de $80 \%$ e especificidade de $21,3 \%$ em identificar aqueles com maior chance de apresentar alterações eletrocardiográficas e que, portanto, deveriam ser avaliados com mais preocupação.

\section{CONCLUSÃO}

No presente estudo, em pacientes portadores da fase crônica da doença de Chagas e eletrocardiograma normal, o intervalo $\mathrm{T} p-\mathrm{Tf}$, corrigido pela frequência cardíaca, não se mostrou marcador prognóstico da manutenção do eletrocardiograma normal. Todavia, quanto maior o intervalo QTc (acima de $372 \mathrm{~ms}$ ) maiores as chances de o paciente apresentar alterações evolutivas do eletrocardiograma.

Trata-se de estudo retrospectivo que analisou as medidas de Tp-Tf e QT manualmente. O alvo foi somente a presença ou não de quaisquer alterações do eletrocardiograma após duas décadas de seguimento, partindo-se de traçado normal, sem avaliar outras manifestações que possam ser compatíveis com cardiopatia. 


\section{REFERÊNCIAS}

1. Vieira, JF, Távora FRF, Sobral GV, Vasconcelos GG, Almeida GPL, Fernandes JR, et al. Chagas cardiomyopathy in Latin America review. Current Cardiology Reports, 2019;21(2):8. https://doi.org/10.1007/s11886-019-1095-y

2. Dias JCP, Ramos Jr. AN, Gontijo ED, Luquetti A, ShikanaiYasuda MA, Coura JR, et al. II Consenso Brasileiro em Doença de Chagas, 2015. Epidemiol Serv Saúde, 2016;25(núm. esp.):7-86. https://doi.org/10.5123/S167949742016000500002

3. Morin DP, Saad MN, Shams OF, Owen JS, Xue JQ, Freddy M, et al. Relationships between the T-peak to T-end interval, ventricular tachyarrhythmia, and death in left ventricular systolic dysfunction. Europace, 2012;14(8):1172-9. https:// doi.org/10.1093/europace/eur426

4. Icli A, Kayrak M, Akilli H, Aribas A, Coskun M, Ozer SF, Ozdemir K. Prognostic value of Tpeak-Tend interval in patients with acute pulmonary embolism. BMC Cardiovascular Disorders, 2015;15(99):1-8. https://doi.org/10.1186/s12872-015-0091-4

5. Panikkath R, Reinier K, Uy-Evanado A, Teodorescu C, Hattenhauer J, Mariani R, et al. Prolonged Tpeak-toTend interval on the resting ECG is associated with increased risk of sudden cardiac death. Circ Arrhythm Electrophysiol, 2011;4(4):441-7. https://doi.org/10.1161/ CIRCEP.110.960658

6. Salles G, Xavier S, Sousa A, Hasslocher-Moreno A, Cardoso C. Prognostic value of QT interval parameters for mortality risk stratification in Chagas': disease results of a long-term follow-up study. Circulation, 2003;108(3):305-12. https:// doi.org/10.1161/01.CIR.0000079174.13444.9C

7. Bradfield J, Woodbury B, Traina M, Hernandez S, Sanchez D, Wachsner R, et al. Repolarization parameters are associated with mortality in Chagas disease patients in the United States. Indian Pacing Electrophysiol J, 2014;14(4):171-80. https:// doi.org/10.1016/s0972-6292(16)30773-2

8. Armaganijan L, Moreira DA, Nolasco de Araújo RR, Puzzi MA, Munhoz FP, Carvalho MJ, et al. The usefulness of T-wave peak to T-wave end interval in identifying malignant arrhythmias in patients with Chagas disease. Hellenic J Cardiol, 2013;54(6):429-34.

9. Pastore CA, Pinho JA, Pinho C, Samesima N, Pereira-Filho HG, Kruse JCL, et al. III Diretrizes da Sociedade Brasileira de Cardiologia sobre análise e emissão de laudos eletrocardiográficos. Arq Bras Cardiol, 2016;106(4 supl.1):123. https://doi.org/10.5935/abc.20160054

10. Sagie A, Larson MG, Goldberg RJ, Bengston JR, Levy D. An improved method for adjusting the QT interval for heart rate (the Framingham Heart Study). Am J Cardiol. 1992;79(7):797801. https://doi.org/10.1016/0002-9149(92)90562-d

11. Hodges M, Salerno D, Erlien D. Bazett's QT correction reviewed: evidence that a linear QT correction for heart is better [abstract]. J Am Coll Cardiol, 1983;1(2):694.

12. Fragata-Filho AA, França FF, Fragata CS, Lourenço AM, Faccini CC, Costa CAJ. Evaluation of parasiticide treatment with benznidazol in the electrocardiographic, clinical, and serological evolution of Chagas disease. PLoS Negl Trop Dis, 2016;10(3):e0004508. https://doi.org/10.1371/journal. pntd.0004508

13. Kors JA, Ritsema van Eck $H J$, van Herpen G. The meaning of the Tp-Te interval and its diagnostic value. J Electrocardiol, 2008;41(6):575-80. https://doi.org/10.1016/j. jelectrocard.2008.07.030

14. Tenekecioglu E, Karaagac K, Yontar OC, Agca FV, Ozluk OA, Tutuncu A, et al. Evaluation of Tp-Te Interval and Tp-Te/ QT ratio in patients with coronary slow flow Tp-Te/QT ratio and coronary slow flow. Eurasian J Med, 2015;47(2):104-8. https://doi.org/10.5152/eurasianjmed.2015.72

15. Youden WJ. Index for rating diagnostic tests. Cancer, 1950;3(1):32-5. https://doi.org/10.1002/10970142(1950) 3:1<32::aid-cncr2820030106>3.0.co;2-3 